\title{
EDUCAÇÃO EM SAÚDE - PROPOSTA DE UTILIZAÇÃO DE UM MODELO NO ENSINO DE CIÊNCIAS
}

\author{
Sheila Soares de Assis ${ }^{1}$, Juliana Novo Borges ${ }^{2}$, Nathália da Rocha Pêssoa Rezende \\ Papoula $^{3}$, Célia Maria da Silva Santiago ${ }^{4}$, Gerlinde Agate Platais Brasil Teixeira ${ }^{5}$ \\ ${ }^{1}$ Universidade Federal Fluminense / Espaço UFF de Ciências/ Sheila.assisbiouff@ gmail.com \\ ${ }^{2}$ Universidade Federal Fluminense / Espaço UFF de Ciências/ junaileve@ hotmail.com \\ ${ }^{3}$ Universidade Federal Fluminense / Espaço UFF de Ciências/ nathipapoula@ig.com.br \\ ${ }^{4}$ Fundação CECCIERJ/ Praça da Ciência Itinerante/ celiamariass@ig.com.br \\ ${ }^{5}$ Universidade Federal Fluminense/ Imunobiologia- Espaço UFF de Ciências/ gerlinde@ vm.uff.br
}

\begin{abstract}
Resumo
Este trabalho visa apresentar a utilização de um modelo didático na abordagem das doenças de veiculação hídrica como ferramenta auxiliar no ensino de ciências mais especificamente no tema de educação em saúde. São apresentados os resultados de uma investigação realizada a partir da execução de uma oficina onde se utiliza um protótipo de uma cidade que reproduz situações facilmente encontradas em regiões urbanas, suburbanas e rurais que foi realizada em eventos de educação não formal. Os resultados mostraram que esta estratégia de ensino em consonância com ideais preconizados pelos temas transversais para a abordagem do tema saúde. Apresenta-se como um material exitoso frente à questão do ensino passivo e não significativo a prática social do aluno que comumente é empregado nas escolas, sejam públicas ou privadas. Vários participantes sugeriram a aplicação desta metodologia no ensino formal.
\end{abstract}

Palavras chave: educação em saúde, ensino de ciências, doenças de veiculação hídrica, ensino formal, ensino não formal.

\begin{abstract}
The aim of this paper is to present the use of a teaching model which addresses waterborne diseases as an auxiliary tool in science teaching related to health education. We present the results of an investigation from the implementation of a workshop where we use the prototype of a city that displays situations easily found in urban, sub urban and rural areas. This activity was presented during science meetings in a non formal format. The results show that this teaching strategy is in consonance with Brazilian educational recommendations of the cross-cutting educational theme related to health issues. It is a successful material to address the commonly applied passive and not significant education, whether in public or private schools, with no connectivity to the student's social practice. Many participants suggested the application of this methodology in formal education.
\end{abstract}

Keywords: health education, science education, water-borne diseases, formally education, not formally education.

\section{INTRODUÇÃO}

A compreensão do conceito de educação em saúde em sua totalidade pressupõe uma análise profunda dos significados atribuídos aos seus dois componentes. No sentido tradicional, educação é o conjunto de atividades que visam transmitir 
conhecimentos, teóricos e práticos, geralmente de forma sistemática (Brasil, 2001). É entendido ainda como um processo de humanização que se dá ao longo de toda a vida, de modos diferentes. Distingui-se como um processo infinito que acontece em múltiplos espaços e situações da vida e está ligada à aquisição e articulação do conhecimento popular e científico, entendido como uma reorganização, incorporação e criação do conhecimento (Rodriguez et. al., 2007). Saúde, por sua vez, adquiriu diversas conceituações ao longo do tempo dentre as mais representativas é àquela dada pela OMS (Organização Mundial da Saúde) onde saúde foi conceituada como "um estado de bem-estar físico, social e mental", conceito considerado utópico e subjetivo que mostra que saúde possui um significado mais amplo do que a mera ausência de doença (Brasil, 2005).

Educação em Saúde, por sua vez é compreendida como qualquer combinação intencional de experiências de aprendizagem com objetivo de facilitar medidas comportamentais ou ações sobre os determinantes sociais da saúde a serem adotadas por pessoas, ou comunidades. Seu processo se distingue de qualquer outro que contenha experiências acidentais de aprendizagem. Na prática, a Educação em Saúde constitui apenas parte das atividades técnicas voltadas para a promoção da saúde. Sendo seu objetivo especifico a organização lógica do componente educativo de programas que se desenvolvem na escola, no local de trabalho, no ambiente clinico e na comunidade, compreendida aqui como contendo populações alvo que não se encontram nas três outras dimensões (Candeias, 1997). É ainda percebida como a expressão do processo teórico-prático planejado e embasada a fim de se obter os resultados desejados que visa integrar vários saberes sejam eles científicos, populares ou do senso comum, proporcionando aos atores envolvidos a percepção crítica e a participação responsável e autônoma. (Diniz et. al., 2009).

No Brasil, ao longo da história, a educação em saúde tem sido marcada por ações voltadas ao combate das doenças infecto-parasitárias nas quais prevalece, ainda hoje, a abordagem acrítica com o predomínio do modelo definido pelo perfil biomédico, sendo hegemônico o saber meramente curativo que não leva a um pensar crítico nem a mudança da realidade. Quanto ao aspecto preventivo incuti apenas normas e padrões comportamentais individuais em substituição à transferência de informação e de esclarecimento sobre os riscos de doença e agravo à saúde (Monteiro et. al., 2006).

$\mathrm{Na}$ escola o indivíduo deve adquirir atitudes e habilidades articuladas às suas experiências de modo que possa reconhecer e expressar suas necessidades, 
possibilitando a oportunidade de refletir e colaborar para possíveis transformações por intermédio de consciência e mudança social (Leonello \& L' Abbate, 2006) A educação em saúde na escola envolve a formação de atitudes e valores que levam o escolar ao comportamento autônomo, revertendo em beneficio a sua saúde e daqueles que estão a sua volta. Neste sentido, a educação não se limita a dar conhecimentos; preocupa-se em motivar o aprendizado, a análise e a avaliação de fontes de informações e torná-lo capaz de adotar práticas comportamentais com base no conhecimento (Marcondes, 1972)

Saúde, na perspectiva educacional é um dos temas transversais explicitados nos Parâmetros Curriculares Nacionais (PCNs) que recomenda que seja abordado de forma ampla com uma construção permanente individual e coletiva e não como uma imagem complementar da doença (Brasil, 1998; Fernandes et. al., 2004). Assim a escola é reconhecida como sendo a instituição voltada ao ensino formal e é elevada ao papel de contribuinte da formação de sujeitos capazes de participar de decisões relativas à saúde individual e coletiva (Fernandes et. al., 2004; Leonello \& L' Abbate, 2006).

$\mathrm{O}$ ensino de Ciências, por conseguinte a abordagem de temas relacionados à saúde no âmbito escolar enfrenta problemas específicos, estes são pautados na necessidade de superação dos modelos tradicionais de ensino, que priorizam a transmissão de conteúdos de forma acrítica, sem evidenciar as dúvidas ou contradições que contribuem para o avanço do conhecimento (Ramos \& Struchiner, 2009). Estratégias interativas de ensino tais como jogos, modelos, dinâmicas entre outras possibilitam que o aluno identifique situações da sua própria vida, possibilitando a geração de diálogos sobre o seu cotidiano, práticas e riscos a qual está sujeito. Enfim, através da utilização adequada de estratégias lúdicas é possível promover uma reflexão sobre sua saúde e sua vida, num contexto de troca com os colegas e o mediador. Em um ambiente onde se usa estes materiais é possível o surgimento de novos conceitos científicos sobre prevenção e cuidado com a saúde, bem como práticas a serem evitadas e soluções coletivas a serem praticadas a partir de movimentos comunitários e de iniciativas da própria escola. (Pimenta et. al., 2006)

Inserido nesta perspectiva os modelos são compreendidos como uma construção, referência, ou estrutura utilizada como uma imagem analógica que permite materializar uma idéia ou conceito, a tornado diretamente assimiláveis (Justina, 2006). O uso de modelos tem sido destacado há muito tempo como uma metodologia importante no ensino de Ciências e Biologia. Estudantes que aprendem com modelos recuperam mais conceitos, memorizam menos informação na forma literal, e geram 
mais soluções criativas para problemas, comparado a estudantes que aprendem sem modelos (Mayer, 1989; Krasilchik, 1996).

Assim os objetivos deste trabalho são apresentar uma forma alternativa para a realização de atividades no âmbito da educação em saúde. Sendo este baseado na construção de modelos com recursos que sejam de baixo custo, reutilizáveis e/ou recicláveis sobre a temática das doenças de veiculação hídrica; verificar a suscetibilidade da população sobre o tema abordado, analisar a utilização do modelo como ferramenta a ser aplicado no âmbito do ensino formal e não formal, bem como o possível estímulo ao senso crítico dos envolvidos de modo que estes vivenciem melhor o tema e possam traçar soluções para os desafios propostos em sua realidade.

\section{MATERIAIS E MÉTODOS}

Entre março e outubro de 2006 foi desenvolvida no Espaço UFF de Ciências uma maquete a ser empregado como ferramenta auxiliar em uma oficina, cuja temática é centrada na abordagem de doenças que possuem influência indireta ou direta da água para sua veiculação. O material aqui destacado foi produzido fazendo uso de duas folhas de isopor sobrepostas, de modo que este possui dimensões de $1,20 \mathrm{~cm}$ referente ao comprimento e $20 \mathrm{~cm}$ de largura. A fim de que fosse conferida maior durabilidade e resistência ao material a base foi revestida com gesso e massa para artesanato. Após a secagem foram utilizados palitos de madeira, latas alumínio provenientes de embalagens de refrigerantes e papelão revestido para a construção de casas e prédios e casas que compõe a maquete. Foi feito uso ainda de parafina em gel para compor o rio que integra o material. Outros objetos como roedores e fezes construídas a base de biscuit, miniaturas de embalagem de produtos industrializados, pequenos brinquedos como carrinhos e bonecos também foram incorporados para que fosse conferida maior similaridade contexto real ao material produzido.

$\mathrm{Na}$ execução da oficina as atividades são iniciadas com a apresentação da maquete, cuja construção foi detalhada anteriormente. Nesta é encontrada uma cidade com diferentes problemas onde se faz uso de uma estratégia de problematização, de modo que o publico identifique e discuta temas como auto cuidado, degradação dos recursos naturais, problemas sociais, cuidado domiciliar e ambiental com vistas à preservação da saúde e prevenção de doenças que podem ser contraídas pela ingestão da água contaminada. Em seguida os visitantes participam de um jogo dinâmico de perguntas e respostas que abrange os diversos aspectos da temática. Os participantes são 
estimulados a refletir de modo que possam adotar um posicionamento crítico frente às diferentes interfaces envolvidas e construam conceitos práticos que podem ser aplicados na sociedade.

Foi aplicado aos participantes um questionário contendo quatro perguntas, (três abertas e uma fechada) cujo objetivo foi investigar o acometimento de doenças típicas do entorno de suas residências e a definição das fontes primárias de informação a cerca da temática. Os participantes foram estratificados quanto à escolaridade As respostas atribuídas às perguntas abertas foram categorizadas, quantificadas e expressos em porcentagem. Analise de variância com pós-teste de Tukey foi utilizada para o tratamento dos dados referentes à idade e escolaridade. Foi adotada significância de $\mathrm{p} \leq$ 0,05 .

A atividade foi aplicada em Niterói, Petrópolis, Duque de Caxias e Maricá entre março de 2007 e outubro de 2008 em eventos de divulgação científica. O público que compõe a amostra deste estudo $(n=130)$ apresenta faixa etária e escolaridade variada, do ensino fundamental incompleto ao ensino superior.

\section{RESULTADOS E DISCUSSÃO}

O público participante da atividade apresenta idade e escolaridade variada distribuída desde o ensino fundamental incompleto ao ensino superior (figura 1). Os integrantes do grupo com ensino médio incompleto apresentaram menor faixa etária $(17,7 \pm 4,2)$, enquanto os do ensino fundamental completo maior idade $(36,2 \pm 11,7)$. A idade média apresenta diferenças significativas quando é comparado ensino fundamental completo e incompleto $(\mathrm{P}<0.01)$, fundamental incompleto e médio incompleto $(\mathrm{P}<0.001)$, fundamental completo e médio completo $(\mathrm{P}<0.01)$ bem como entre médio incompleto e superior $(\mathrm{P}<0.05)$.

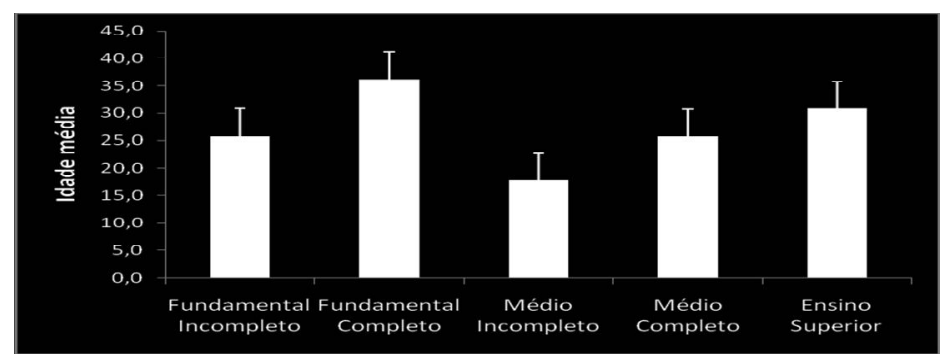

Figura 1: Distribuição média da idade dos participantes

A maioria dos indivíduos declarou que na comunidade onde residem existe algum dos problemas mostrados na maquete, embora alguns não fossem capazes de 
reconhecê-los. Para todos que responderam sim nesta questão foi solicitado que indicassem quais as situações problema que foram identificadas na maquete utilizada na oficina se assemelhava com as encontradas no entorno de sua residência (figura 2).

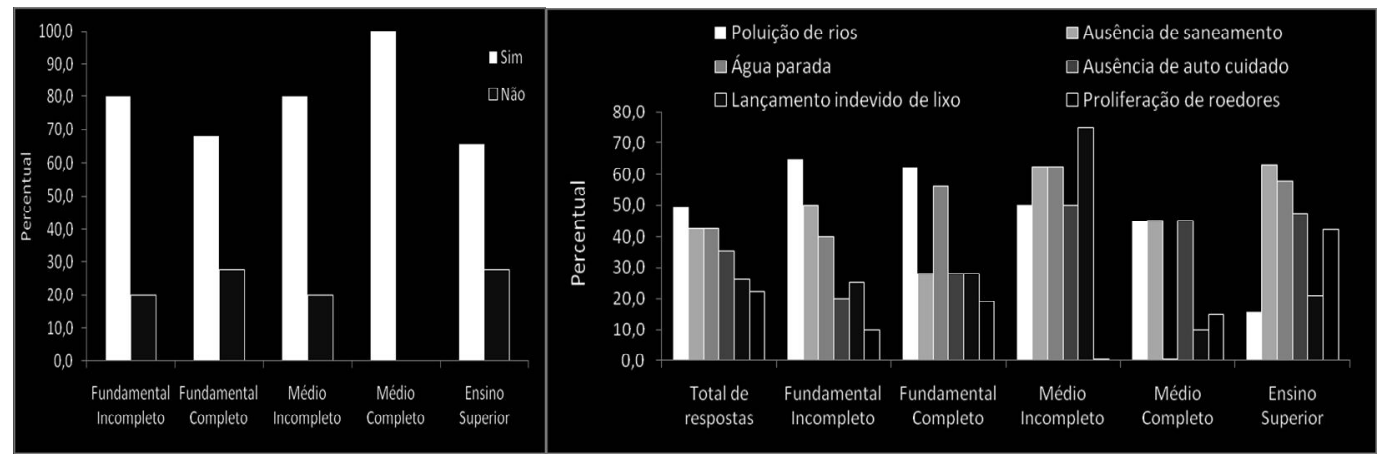

Figura 2: Percentual de participantes que a partir do modelo, identificaram, ou não problemas no entorno de suas residências (Esquerda) e percentual de aspectos especificados (Direita) distribuídos de acordo com a escolaridade dos participantes.

A adoção de estratégias para a Educação em Saúde nas quais o aluno consegue identificar sua realidade no objeto de estudo apresenta-se como uma metodologia capaz de proporcionar uma formação crítica onde o discente não é um mero receptor. Se explorada de forma estruturada, pode constituir uma estratégia de aprendizagem significativa, possibilitando a interação entre o "novo" conhecimento e a rede conceitual que o aluno já possui (Farias \& Bandeira, 2009).

A poluição dos rios foi o problema apontado com maior freqüência (50\%) confirmando dados da literatura que indicam que a degradação dos recursos naturais vem aumentando de forma alarmante, principalmente, as fontes hídricas. Como conseqüência águas subterrâneas vêm sendo utilizados cada vez mais para o consumo humano. No entanto, o grande problema é a contaminação destas por microrganismos patogênicos (Rohden, 2009). As duas principais categorias de riscos à saúde relacionados à contaminação da água são por: 1) agentes biológicos (vírus, bactérias e parasitas) por ingestão de água contaminada, através de contato direto ou por meio de insetos vetores que necessitam da água em seu ciclo biológico; 2) poluentes químicos em geral decorrentes de efluentes de esgotos industriais (Aguila et. al., 2000).

Em acordo com os dados do Instituto Brasileiro de Geografia e Estatística (IBGE, 2000) que mostra que no sudeste 7\% dos municípios não tem coleta, $60 \%$ só coletam e apenas $33 \%$ coletam e tratam o esgoto, a ausência de saneamento básico foi o segundo problema mais frequente que é considerado um importante índice de qualidade 
de vida da população, já que sua existência traz mais conforto, melhores condições de saúde e preservação da qualidade do meio ambiente (Souza \& Silva Junior, 2004).

Outra situação presente no entorno das residências de todos os participantes é o lançamento indevido de lixo. O grupo que mais destaca esta situação é o de Ensino médio incompleto. A ausência do serviço de coleta de lixo é mais frequiente em regiões periféricas das grandes cidades já que a ocupação desordenada dificulta a coleta. Estudos têm apontado para uma possível associação entre o manejo inadequado de lixo domestico e o aumento de eventos de diarréia e parasitoses intestinais (Rêgo et. al. 2002) e que a falta de saneamento básico constitui fator essencial para a proliferação de roedores e estes por sua vez são vetores significativos da leptospirose (Almeida et. al., 1994). Mais uma vez nossos entrevistados estão atentos, muitos apontam este aspecto.

A adoção de medidas de higiene pessoal regular constitui uma importante forma de prevenção de doenças infecciosas muitas das quais são de veiculação hídrica (Brasil, 2005) A ausência de auto cuidado, relacionada à carência de fatores referentes à higiene pessoal foi identificado por todas as categorias.

Para alcançar a meta de saúde para todos faz se necessário que seja desenvolvida a atenção primária, aliada a uma educação voltada às necessidades e possibilidades da comunidade. Assim, faz-se necessário educar as grandes massas por meio de medidas educativas e sanitárias, de modo a promover atividades em favor da saúde, estimulando a eliminação de mosquitos, a desratização, a ingestão de água potável, a mudança de hábitos de higiene, entre outras. (Rodriguez et. al., 2007, Campos, 2001).

Em relação às doenças de veiculação hídrica abordadas na atividade os 74,6\% dos participantes disseram que eles próprios ou algum de seus familiares já foram acometidos por dengue, verminoses, rotavirose, leptospirose ou hepatites (Figura3).

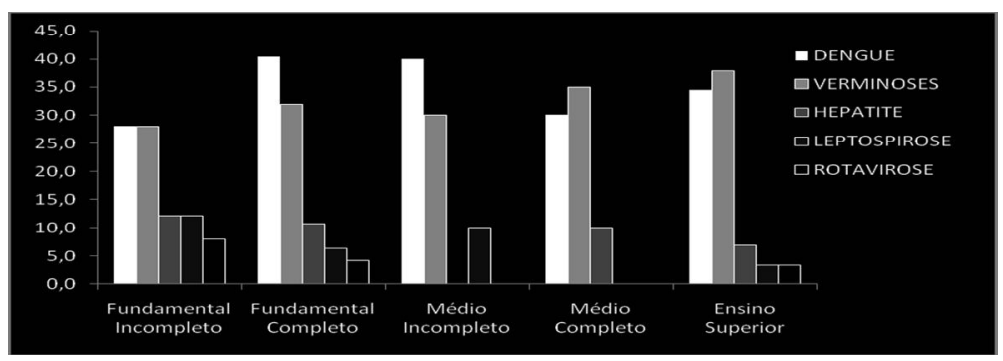

Figura 3: Percentual de doenças citadas como de veiculação hídrica estratificado por escolaridade

Entre as doenças de maior prevalência a dengue e as parasitoses ganharam destaque em todos os grupos. Originária do sudeste da Ásia a dengue é uma doença viral de caráter endêmico-epidêmico, transmitida principalmente pelo mosquito Aedes 
aegypti, que ocorre em sua maioria em climas tropicais e subtropicais, sendo hoje uma das mais importantes doenças tropicais que afligem as Américas. É considerada uma grave ameaça à saúde de milhões de pessoas. O grande desafio, nas ações educativas promovidas por setores da educação e da saúde, para controlar a doença, é esclarecer a população uma vez que o controle do vetor só é possível quando há o envolvimento da comunidade (Medronho, 2008; Brasil, 2009).

As enteroparasitoses ou parasitoses intestinais ocorrem em decorrentes da infecção por protozoários e/ou helmintos decorrente das más condições de vida da população. Constituem um problema de saúde pública de alta incidência mundial (Afiune et. al., 2009; Siwila et. al., 2009). A transmissão das parasitoses intestinais ocorre em sua maior parte por via oral passiva, com a ingestão de água e/ou alimentos contaminados, sendo a sua prevalência associada a áreas que se apresentam com condições higiênico-sanitárias precárias vinculadas a falta de tratamento adequado da água de consumo e do esgoto doméstico (Cardoso, 1995). Entre as medidas efetivas para a redução da infecção parasitária estão inclusas a educação para a prevenção e o acesso universal ao sistema de saúde (Melo et. al., 2004).

Em menor número foram destacadas: Hepatite, Leptospirose e Rotavirose. As hepatites virais apresentam distribuição universal e magnitude variável de acordo com a região do país, sendo as hepatites A e E mais prevalentes onde as condições sanitárias e socioeconômicas são precárias. (Brasil, 2004).

A leptospirose é uma zoonose de caráter endêmico, podendo apresentar-se na forma de surto ou epidemia sob certas condições climáticas, ambientais e de infraestrutura sanitária. No Brasil, a maior parte dos casos está ligada à infra-estrutura sanitária domiciliar. Enchentes decorrentes de chuvas fortes também são fatores de contato do homem com água e lama contaminadas pela urina de roedores (Brasil, 2004).

Através de uma questão fechada, onde mais de uma alternativa podia ser respondida, foram investigadas quais as principais fontes de informação a respeito da temática abordada na oficina (Figura 4).

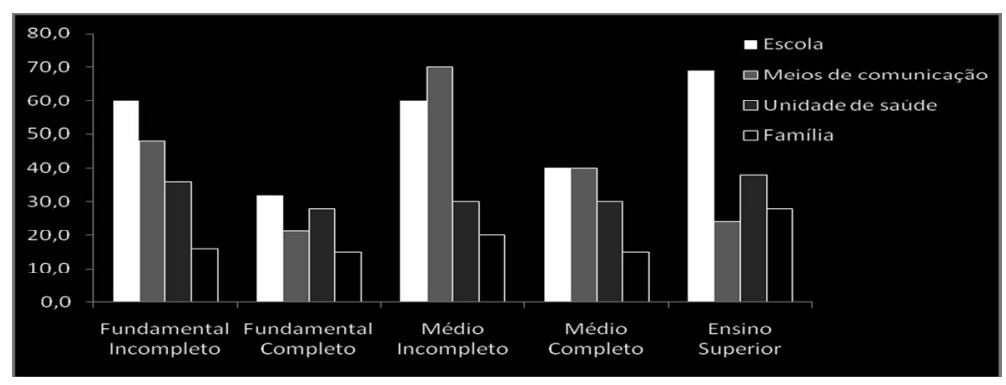


Figura 4 principais fontes de informação sobre questões de saúde estratificadas por escolaridade

Com exceção dos indivíduos do ensino médio, todos apresentaram a escola como principal fonte de informação. Estes, em sua maioria, apontam os meios de comunicação como sendo a sua principal fonte bibliográfica havendo uma valorização dos meios de comunicação disponíveis em nossa sociedade principalmente a internet e televisão. Este dado é semelhante ao de autores como Mello \& Wiggers. (2008) e Pontes et. al. (2009) que investigaram a fonte de diferentes tipos de informações entre jovens.

Outro item apontado como fonte de informação relacionada aos aspectos abordados na oficina são as unidades de saúde. É importante haver uma interação entre a escola e as equipes de saúde que devem articular suas estratégias para promoverem a educação em saúde (Leonello \& L' Abbate, 2006 Rodrigues, 2007)

A última etapa foi à avaliação das atividades. Foram estabelecidas cinco categorias a partir das respostas dos participantes que de acordo com o grau de escolarização valorizou diferentes aspectos (Figura 5). A criatividade foi valorizada pelos participantes com Ensino fundamental enquanto que os alunos com Ensino médio valorizaram a semelhança sugeriram que este é um bom modelo para ser utilizado no ensino básico. É importante destacar que este grupo é composto principalmente por professores do ensino básico, muitos atuantes no primeiro ciclo do ensino fundamental.

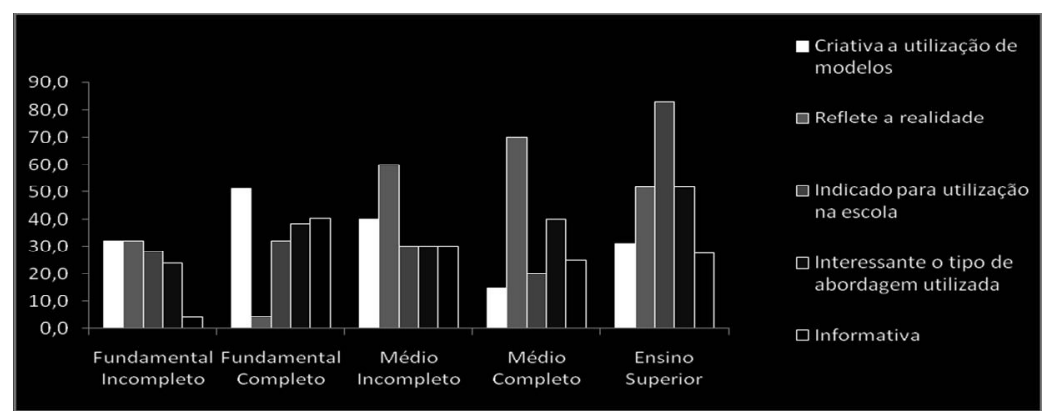

Figura 5: Avaliação das atividades realizadas categorizadas por escolaridade

Os problemas representados na maquete, que estão amplamente distribuídos em todas as regiões brasileiras, foram adequadamente dimensionados uma vez que a categoria "reflete a realidade" foi verificada em todas as categorias. Assim a utilização desta estratégia como ferramenta didática permite a identificação pelos participantes de conteúdos escolares e científicos no seu cotidiano. Pudemos observar que a utilização de uma ferramenta que aborda temas essenciais de educação em saúde de forma lúdica permite que o aprendizado ocorra de modo mais leve, e a assimilação de conceitos se dá 
através da construção / reconstrução de conceitos críticos que lhe permitam preservar a saúde. O público ainda aponta que a utilização de modelos para abordagem desta temática é uma estratégia criativa e interessante. Acreditamos que isso se deve ao fato do material concreto ser capaz de prender a atenção dos participantes de modo que estes visualizam o que lhes tá sendo falado. Neste sistema a escola tem papel fundamental. Embora a atividade tenha sido empregada em ambientes de ensino não formal foi reconhecida sua importância no meio escolar e que esta metodologia pode contribuir de forma significativa para que sejam atingidos os ideais preconizados pelos PCNs.

Sugerimos que os modelos destinados ao ensino de Ciências e Biologia sejam estimulados pelos docentes e construídos pelos próprios alunos, garantindo que os alunos possam exercer e estimular seu potencial criativo, construindo novos conceitos de forma condizente com uma prática social crítica, os tornado atores ativos na transformação de sua realidade. Destacamos ainda que a construção de uma sociedade mais saudável e mais justa não pode ignorar o papel estratégico da educação em saúde que tem se defrontado com novos desafios, como os de produzir em ritmo compatível com as transformações da sociedade recursos pedagógico adequados às necessidades de um público em constante transformação cultural e comportamental.

\section{CONCLUSÃO}

Foi viável a construção de um material educativo de baixo custo e com grande apelo visual que refletisse de fato a realidade de degradação de recursos hídricos, ausência de auto cuidado, proliferação de roedores e deficiência quanto aos serviços de coleta de esgoto e lixo domestico comumente encontrada no ambiente rural e urbano.

Percebemos a vulnerabilidade a qual os diferentes sujeitos que integraram a amostra, independente da escolaridade, estão submetidos frente às doenças correlacionadas com água. Concluímos, então que a utilização de um modelo concreto e uma dinâmica como estratégia interativa foram eficazes na abordagem da temática das doenças de veiculação hídrica em uma atividade de educação em saúde desenvolvida em espaços de educação não formal.

\section{REFERÊNCIAS}

Afiune, L. A. F.; Ribeiro, H. S., Costa, C. V. Correlação entre condições de saneamento básico e parasitoses intestinais em duas creches, uma no município de Barra das Garças - e a outra em Pontal do Araguaia - MT. Anais do XI - EREGEO - Simpósio

Regional de Geografia. Goiás, 2009. 
Aguila, P. S.; Roque, O. C. C.; Miranda, C. A. S.; Ferreira, A. P. Avaliação da qualidade de água para abastecimento público do Município de Nova Iguaçu. Cadernos de Saúde Pública. Rio de Janeiro, 16 (3): 791-798, 2000.

Almeida, L.P.; Martins, L.F.S.; Brod, C.S.; Germano, P.M.L. Levantamento soroepidemiológico de leptospirose em trabalhadores do serviço de saneamento ambiental em localidade urbana da região sul do Brasil. Revista de Saúde Pública. São Paulo, v. 28 (n.1): 76-81, 1994.

Brasil. Parâmetros Curriculares Nacionais. Ministério da Educação. Brasília, 1998.

Brasil. INEP - Instituto Nacional de Estudos e Pesquisas Educacionais Anísio Teixeira. 2001. Disponível em: http://www.inep.gov.br/pesquisa/thesaurus/. Acessado em 21/01/2010.

Brasil. Situação e controle das doenças transmissíveis no Brasil. Saúde Brasil - Uma análise da situação de saúde. Ministério da Saúde. Brasília, 2004.

Brasil. Plano de Contingência de Vigilância em Saúde Frente a Inundações. Ministério da Saúde. Brasília, 2005.

Brasil. Educação que produz saúde. Ministério da Saúde. Brasília, 2005.

Brasil, Casos de dengue caem 47,9\% em 2009. Ministério da Saúde. Disponível em: <http://portal.saude.gov.br> Acesso em 29/09/2009.

Campos, F.E., Ferreira, J.R., Feuerwerker, L., Sena, R.R., Campos, J.J.B., Cordeiro, H., Cordoni Jr, L. Caminhos para Aproximar a Formação de Profissionais de Saúde das Necessidades da Atenção Básica. Revista Brasileira de Educação Médica. v. 25(2):53-59, 2001.

Candeias, N. M. F. Conceitos de educação e promoção em saúde. Revista de Saúde Pública. São Paulo, v. 31(2):209-213, 1997.

Cardoso, G. S; Santana, A. D. C.; Aguiar, C. P. Prevalência e aspectos epidemiológicos da giardíase em creches no município de Aracaju, SE, Brasil. Revista da Sociedade Brasileira de Medicina Tropical. Minas Gerais, v. 28(1): 25-31, 1995.

Diniz, M.C.P., Figueiredo, B.G., Schall, V.T. Hortênsia de Hollanda: a arte da educação em saúde para a prevenção e controle das endemias no Brasil. História, Ciências, Saúde - Manguinhos. v.16(2): 533-548 2009

Farias, E. M.; Bandeira, K. S. O uso das analogias no ensino de ciências e de biologia. Ensino, Saúde e Ambiente. v. 2(3): 60 -71, 2009.

Fernandes, C.A.M; Bacarin, A.C.B.P.; Borges, G.F.; Nardo, C.C.S.; Nardo Júnior,N.; Tirapegui, J. Promoção da saúde na escola: uma proposta emergente e Emergencial. Revista APADEC. 8 (supl.):621-626, 2004.

IBGE- Instituto Brasileiro de Geografia e Estatística. Pesquisa Nacional de Saneamento Básico. Rio de Janeiro, 2000.

Justina, L.A.D.; Faria, M.R. A utilização de modelos didáticos no ensino de Genética exemplo de representação de compactação do DNA eucarioto. Arquivo Mudi v. 10(2):35-40, 2006.

Krasilchik, M. Prática de Ensino de Biologia. Ed. Harbra. São Paulo, 1996. 
Leonello, V.M.; L' Abbate, S. Educação em saúde na escola: uma abordagem do currículo e da percepção de alunos de graduação em pedagogia. Interface-

Comunicação, Saúde e Educação. v.10 (19): 149-166, 2006.

Marcondes, R.S. Educação em saúde na escola. Revista de Saúde Pública. v.6(1):8996, 1972.

Mayer, R.E. Techniques that help readers build mental models. Review of Education Research. V.59(1):43-64,1989.

Medronho, R.A. Dengue no Brasil: desafios para o seu controle Cadernos de Saúde Pública. v.24(5):948-949, 2008.

Melo, M.C.B.; Klem, V.G.Q.; Mota, J.A.C.; Penna, F.J. Parasitoses Intestinais. Revista Médica de Minas Gerais. v.14, (Sup.1):3-12 ; 2004;.

Mello, H.D.; Wiggers, I.D. Representações e usos da Internet: um estudo de recepção com adolescentes. Revista Iberoamericana de Educación. v. 45(2):1-14, 2008.

Monteiro, S.; Vargas, E.; Cruz, M. Desenvolvimento e uso de tecnologias educacionais no contexto da AIDS e da saúde reprodutiva: Reflexões e perspectivas. In: Monteiro, S. e Vargas, E.(Orgs.) Educação, Comunicação e Tecnologia Educacional: interfaces com o campo da saúde. Rio de Janeiro, FIOCRUZ, p. 27-48, 2006.

Pimenta, D.N.; Leandro, A.M.S.; Schall, V.T. Experiências de desenvolvimento e avaliação de materiais educativos sobre saúde: Abordagens sócio-históricas e contribuições da antropologia visual. In: In: Monteiro, S. e Vargas, E. (Orgs.)

Educação, Comunicação e Tecnologia Educacional: interfaces com o campo da saúde. FIOCRUZ,87-112, 2006.

Pontes, T. E.; Costa, T. F.; Marum, A. B. R. F.; Brasil, A. L. D.; Taddei, J. A. A. C. Orientação nutricional de crianças e adolescentes e os novos padrões de consumo: propagandas, embalagens e rótulos. Revista Paulista de Pediatria. São Paulo, v. 27 (1):99-105, 2009.

Ramos, P.; Struchiner, M. Concepções de educação em pesquisas sobre materiais informatizados para o ensino de ciências e de saúde. Ciência e Educação. São Paulo, 15 (3): 13-27, 2009.

Rêgo, R. C. F.; Barreto, M. L.; Killinger, C. L. O que é lixo afinal? Como pensam mulheres residentes na periferia de um grande centro urbano. Cadernos de Saúde Pública. Rio de Janeiro, v. 18 (6): 1583-1591, 2002.

Rodriguez, C. A., Kolling, M. G., Mesquita, P. Educação em Saúde: um binômio que merece ser resgatado. Revista Brasileira de Educação Médica. 31 (1): 3-4 2007.

Rohden, F.; Rossi, E. M.; Scapin, D.; Cunha, F. B.; Sardiglia, C. U. Monitoramento microbiológico de águas subterrâneas em cidades do Extremo Oeste de Santa Catarina. Ciência \& Saúde Coletiva. Rio de Janeiro, v.14 (6): 2199-2203, 2009.

SEDESC-RJ. Relatório de Casos De Dengue - 2009. Disponível em: <http://www.saude.rj.gov.br/Docs/Acoes/dengue/Relat\%C3\%B3rio\%20de\%20Casos\% 20de\%20Dengue\%20_02-07-2009\%20-\%2016h00m_.pdf>. Acesso em: 30/09/2009.

Siwila, J.; Phiri, I.G.K., Enemark, H. L., Nchito, M.; Olsen, A. Intestinal helminthes and protozoa in children in pre-schools in Kafue district, Zambia. Transctions of the Royal Society of Tropical Medicine and hygiene. London,v. 104 (2): 33-52, 2009. 
Souza, R. F. P.; Silva Junior, A. G. . Poluição Hídrica e Qualidade de Vida: O caso do Saneamento Básico no Brasil. In: XIII Congresso da Sober. Cuiabá, 2004. 